\title{
A Robust Watermarking Technique based on Nonsubsampled Contourlet Transform and SVD
}

\author{
C.Venkata Narasimhulu \\ Professor \\ Dept of ECE, HIET, \\ Hyderabad, India
}

\author{
K.Satya Prasad \\ Professor \\ Dept of ECE, JNTU \\ Kakinada, India
}

\begin{abstract}
The paper proposes a novel robust watermarking technique based on newly introduced Nonsubsampled contourlet transform(NSCT) and singular value decomposition(SVD) for multimedia copyright protection. The NSCT can give the asymptotic optimal representation of the edges and contours in image by virtue of the characteristics of good multi resolution shift invariance and multi directionality. After decomposing the host image into sub bands, we choose the low frequency directional sub band and apply singular value decomposition. The singular values of the original image are then modified by the singular values of nonsubsampled contourlet transformed visual grayscale logo watermark image. This hybrid approach improves the performance of the watermarking technique compared to earlier techniques. Experimental results shows that the hybrid technique is resilient to various linear and non linear filtering ,JPEG compression, JPEG2000 compression, Histogram equalization, Grayscale inversion, Contrast adjustment, gamma correction, alpha mean ,cropping ,Gaussian noise, scaling etc.
\end{abstract}

\section{KEYWORDS}

Image watermarking, nonsubsampled contourlet transform, SVD, visual watermark logo.

\section{INTRODUCTION}

Rapid growth in digital technique and internet usage has created a new set of challenging problems such as copyright protection, authentication and content integrity verification of the digitized properties. Over the last few years, watermarking is popularly known as a potential solution to address these problems through invisible insertion of auxiliary message (logo/symbol) called watermark in digital data [1] This insertion data can be later extracted from or detected in the multimedia to make an assertion about the data. Digital watermarks remain intact under transmission/transformation, allowing us to protect our ownership rights in digital form. Absence of watermark in a previously watermarked image would lead to the conclusion that the data content has been modified. A watermarking algorithm consists of watermark structure, an embedding algorithm and extraction or detection algorithm. In multimedia applications, embedded watermark should be invisible, robust and have a high capacity. Invisibility refers to degree of distortion introduced by the watermark and its affect on the viewers and listeners. Robustness is the resistance of an embedded watermark against intentional attack and normal signal processing operations such as noise, filtering, rotation, scaling, cropping and lossey compression etc. Capacity is the amount of data can be represented by embedded watermark [2], [3]

Watermarking techniques may be classified in different ways. The classification may be based on the type of watermark being used, i.e., the watermark may be a visually recognizable logo or sequence of random numbers. A second classification is based on whether the watermark is applied in the spatial domain or the transform domain. In spatial domain, the simplest method is based on embedding the watermark in the least significant bits (LSB) of image pixels. However, spatial domain techniques are not resistant enough to image compression and other image processing operations.

Transform domain watermarking schemes such as those based on the discrete cosine transform (DCT), the discrete wavelet transform (DWT), contourlet transforms along with numerical transformations such as Singular value Decomposition (SVD) and Principle component analysis (PCA) typically provide higher image fidelity and are much robust to image manipulations.[4]Of the so far proposed algorithms, wavelet domain algorithms perform better than other transform domain algorithms since DWT has a number of advantages over other transforms including time frequency localization, multi resolution representation, superior HVS modeling, and linear complexity and adaptively and it has been proved that wavelets are good at representing point wise discontinuities in one dimensional signal. However, in higher dimensions, e.g. image, there exists line or curve-shaped discontinuities. Since, 2D wavelets are produced by tensor products of 1D wavelets; they can only identify horizontal, vertical, diagonal discontinuities (edges) in images, ignoring smoothness along contours and curves. Curvelet transform was defined to represent two dimensional discontinuities more efficiently, with least square error in a fixed term approximation. Curvelet transform was proposed in continuous domain and its discretisation was a challenge when critical sampling is desired. Contourlet transform was then proposed by DO and Vetterli as an improvement of Curvelet transform. The Contourlet transform is a directional multi resolution expansion which can represents images contains contours efficiently. The CT employs Laplacian pyramids to achieve multi resolution decomposition and directional filter banks to achieve directional decomposition [4],[5],[6] Due to down sampling and up sampling, the Contourlet transform is Shift variant. However shift invariance is desirable in image analysis applications such as edge detection, Contour characterization, image enhancement [7] and image watermarking. Here, we present a NonSubsampled Contourlet transform (NSCT) [8] which is shift invariant version of the contourlet transform. The NSCT is built upon iterated nonsubsampled filter banks to obtain a shift invariant image representation.

In all above transform domain watermarking techniques including NSCT the watermarking bits would be directly embedded in the locations of sub band coefficients. Though here the visual of perception of original image is preserved, the watermarked image when subjected to some intentional attacks like compression the watermark bits will get damaged. 
Coming to the spatial domain watermarking using numerical transformation like SVD (Gorodetski [9], liu et al [10]) they provide good security against tampering and common manipulations for protecting rightful ownership. But these schemes are non adaptive, thus unable to offer consistent perceptual transparency of watermarking of different images [11]To provide adaptive transparency, robustness to the compressions and insensitivity to malicious manipulations, we propose a novel image hybrid watermarking scheme using NSCT and SVD.

In this paper, proposed method is compared with another which is based on discrete wavelet transform and singular value decomposition (DWT-SVD).The peak signal noise ratio (PSNR) between the original image and watermarked image and the normalized correlation coefficients (NCC) after different attacks were calculated. The results show high improvement detection reliability using proposed method.The rest of this paper is organized as follows. Section 2 describes the Nonsubsampled contourlet transform, section 3 describes singular value decomposition, section 4 illustrates the details of proposed method, in section 5 experimental results are discussed without and with attacks, conclusion and future scope are given in section 6 .

\section{NONSUBSAMPLED CONTOURLET TRANSFORM}

The nonsubsampled contourlet transform is a new image decomposition scheme introduced by Arthur L.Cunha, Jianping Zhou and Minh N.Do [12] NSCT is more effective in representing smooth contours in different directions of in an image than contourlet transform and discrete wavelet transform. The NSCT is fully shift invariant, Multi scale and multi direction expansion that has a fast implementation. The NSCT exhibits similar sub band decomposition as that of contourlets, but without down samplers and up samplers in it. Because of its redundancy, the filter design problem of nonsubsampled contourlet is much less constrained than that of contourlet [12],[13],[14] The NSCT is constructed by combining nonsubsampled pyramids and nonsubsampled directional filter bank as shown in Figureure 1.The nonsubsampled pyramid structure results the multi scale property and nonsubsampled directional filter bank results the directional property.

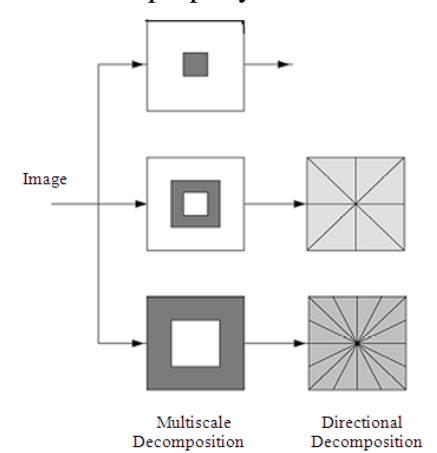

(a)

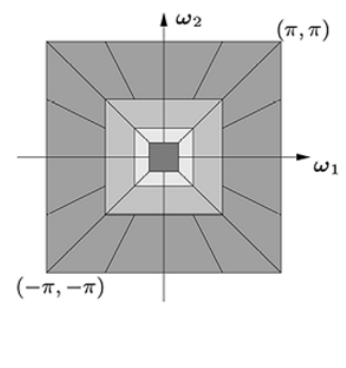

(b)
Figure 1 The nonsubsampled contourlet transform (a) nonsubsampled filter bank structure that implements the NSCT. (b) Idealized frequency partitioning obtained with NSCT

\subsection{Nonsubsampled pyramids}

The nonsubsampled pyramid is a two channel nonsubsampled filter bank as shown in Figureure 2(a).The $\mathrm{H}_{0}(\mathrm{z})$ is the low pass filter and one then sets $\mathrm{H}_{1}(\mathrm{z})=1-\mathrm{H}_{0}(\mathrm{z})$ and corresponding synthesis filters $\mathrm{G}_{0}(\mathrm{z})=\mathrm{G}_{1}(\mathrm{z})=1$. The perfect reconstruction condition is given by Bezout identity[7],[8],[12]

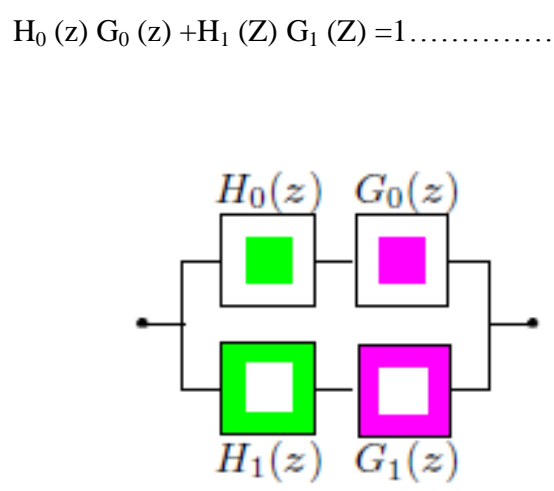

(a)

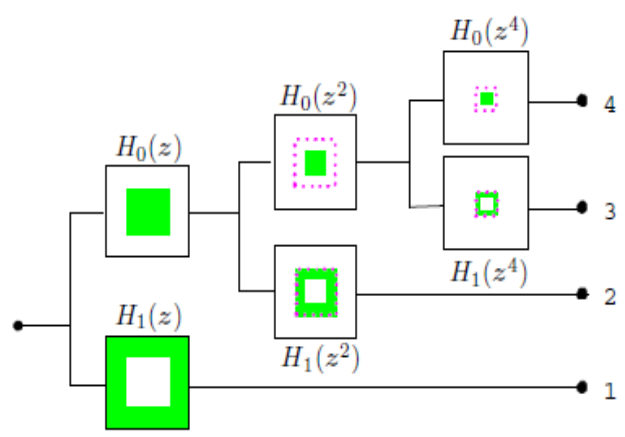

(b)

Figure 2 Nonsubsampled pyramidal filters (a). Ideal frequency response of nonsubsampled pyramidal filter (b).The cascading analysis of three stages nonsubsampled pyramid by iteration of two channels nonsubsampled filter banks.

Multi scale decomposition is achieved from nonsubsampled pyramids by iterating the nonsubsampled filter banks. The next level decomposition is achieved by up sampling all filters by 2 in both dimensions. The complexity of filtering is constant whether the filtering is with $\mathrm{H}(\mathrm{z})$ or an up sampled filter $\mathrm{H}\left(\mathrm{z}^{\mathrm{m}}\right)$ computed using ' a trous ' algorithm [15] The cascading of three stage analysis part is shown in Figureure2 (b).

\subsection{Nonsubsampled directional Filter Banks}

The directional filter bank (DFB) [16] is constructed from the combination of critically-sampled two-channel fan filter banks and resampling operations. The outcome of this DFB is a treestructured filter bank splitting the 2-D frequency plane into wedges. The nonsubsampled directional filter bank which is shift invariant is constructed by eliminating the down and up samplers in the DFB.[13]The ideal frequency response of nonsubsampled filter banks is shown in Figureure3 (a) 


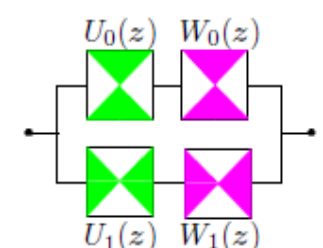

(a)

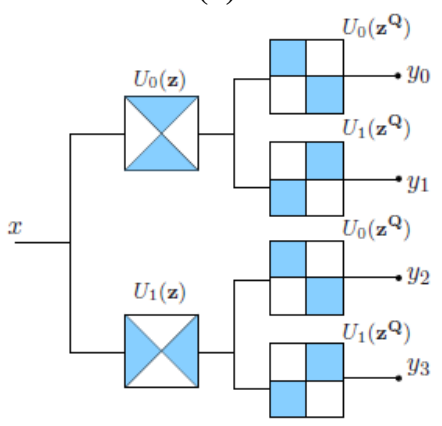

(b)

\section{Figure 3 Nonsubsampled directional filter bank (a) idealized frequency response of nonsubsampled directional filter bank.(b) The analysis part of an iterated nonsubsampled directional bank.}

To obtain multi directional decomposition, the nonsubsampled DFBs are iterated. To obtain the next level decomposition, all filters are up sampled by a quincunx matrix given by [7],[8]

$$
Q=\left(\begin{array}{rr}
1 & 1 \\
1 & -1
\end{array}\right) \text {.............. } 2
$$

The analysis part of an iterated nonsubsampled filter bank is shown in Figure 3(b).

\section{SINGULAR VALUE DECOMPOSITION}

Singular value decomposition (SVD) is a popular technique in linear algebra and it has applications in matrix inversion, obtaining low dimensional representation for high dimensional data, for data compression and data denoising. If A is any $\mathrm{N} \times \mathrm{N}$ matrix, it is possible to find a decomposition of the form

$$
\begin{aligned}
& \mathrm{A}=\mathrm{USV}^{\mathrm{T}} \\
& A=\left[\begin{array}{llll}
u_{1} & u_{2} & \ldots & u_{n}
\end{array}\right]\left(\begin{array}{llll}
\lambda_{1} & & & \\
& \lambda_{2} & & \\
& & \cdot & \\
& & & .
\end{array}\right)\left[\begin{array}{lll}
v_{1} & v_{2} \ldots & v_{n}
\end{array}\right]^{T}
\end{aligned}
$$

Where $\mathrm{U}$ and $\mathrm{V}$ are orthogonal matrices of order $\mathrm{N} \times \mathrm{N}$ and $\mathrm{N}$ $x \mathrm{~N}$ such that $\mathrm{U}^{\mathrm{T}} \mathrm{U}=\mathrm{I}, \mathrm{V}^{\mathrm{T}} \mathrm{V}=\mathrm{I}$, and the diagonal matrix $\mathrm{S}$ of order $\mathrm{N} x \mathrm{~N}$ has elements $\lambda_{\mathrm{i}} \quad(\mathrm{i}=1,2,3, . . \mathrm{n})$, I is an identity matrix of order $\mathrm{N} x \mathrm{~N}$.
The diagonal entries are called singular values of matrix A, the columns of $U$ matrix are called the left singular values of $\mathrm{A}$, and the columns of $\mathrm{V}$ are called as the right singular values of A. [4]

The general properties of SVD are [2], [4], [10]

a) Transpose: $A$ and its transpose $A^{T}$ have the same nonzero singular values.

b) Flip: A, row-flipped Arf, and column-flipped Acf have the same non-zero singular values.

c) Rotation: $A$ and $\operatorname{Ar}$ ( $A$ rotated by an arbitrary degree) have the same non-zero singular values.

d) Scaling: $B$ is a row-scaled version of $A$ by repeating every row for $L 1$ times. For each non-zero singular value $\lambda$ of $A, B$ has $\sqrt{L 1 \lambda}$. $C$ is a column-scaled version of $A$ by repeating every column for $L 2$ times. For each nonzero singular value $\lambda$ of $A, C$ has $\sqrt{ } L 2 \lambda$. If $D$ is row-scaled by $L 1$ times and columnscaled by $L 2$ times, for each non-zero singular value $\lambda$ of $A, D$ has $\sqrt{ } L 1 L 2 \lambda$.

e) Translation: $A$ is expanded by adding rows and columns of black pixels. The resulting matrix $A e$ has the same Nonzero singular values as A.

The important properties of SVD from the view point of image processing applications are:

1. The singular values of an image have very good stability i.e. When a small perturbation is added to an image, their singular values do not change significantly.

2. Singular value represents intrinsic algebraic image properties. [2],[3],[4],[10],[17],[18]

Due to these properties of SVD, in the last few years several watermarking algorithms have been proposed based on this technique. The main idea of this approach is to find the SVD of a original image and then modify its singular values to embedded the watermark. Some SVD based algorithms are purely SVD based in a sense that only SVD domain is used to embed watermark into original image. Recently some hybrid SVD based algorithms have been proposed where different types of transform domains including discrete cosine transform (DCT), discrete wavelet transform (DWT), Contourlet transform (CT) etc are used to embed watermark into original image. Here the proposed scheme uses nonsubsampled contourlet transform (NSCT) along with SVD for watermarking to obtain better performance compared to existing hybrid algorithms.

\section{PROPOSED ALGORITHM}

In this paper, NSCT and SVD based hybrid technique is proposed for watermarking of gray scale watermark image on gray scale original image. The robustness and perceptuality of watermarked image is tested with two quantifiers such as PSNR and NCC. It is investigated whether the NSCT-SVD advantages over DWT-SVD with their extra features would provide any significant in terms of watermark robustness and invisibility.4.1, 4.2 explain the watermark embedding and extraction algorithm [2],[3],[4],[19] 


\subsection{Watermark Embedding Algorithm}

The proposed watermark embedding algorithm is shown in Figure 4 . The steps of watermark embedding algorithm are as follows.

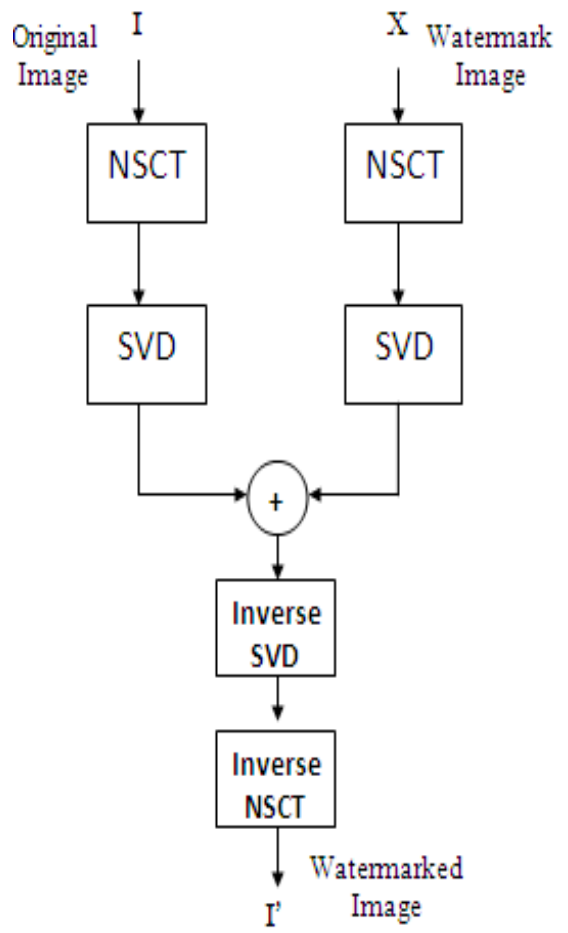

Figure 4. Watermark Embedding Algorithm

Step1: Apply NSCT to the original image to decompose into sub bands.

Step2: Apply SVD to low frequency sub band of NSCT of original image.

Step3: Apply NSCT to gray scale logo watermark to decompose into sub bands.

Step4: Apply SVD to low frequency sub band of NSCT of gray scale logo watermark image.

Step5: Modify the singular values of original image with the singular values of gray scale image watermark.

i.e. $\quad \lambda_{\mathrm{I}},=\lambda_{\mathrm{I}}+\alpha \lambda_{\mathrm{W}}$.,

Where $\alpha$ is scaling factor, [4] $\lambda_{\mathrm{I}}$ is singular value of original image, $\lambda_{\mathrm{W}}$ is singular value of gray scale logo watermark and $\lambda_{\mathbf{I}}$, becomes singular value of watermarked image.

Step6: Apply inverse SVD of transformed original image with modified singular values in step 5 .

Step7: Apply inverse NSCT using the modified coefficients of the low frequency bands to obtain the watermarked image.

\subsection{Watermark Extraction Algorithm}

The watermark extraction algorithm is shown in Figureure 5. The Steps of watermark extraction algorithm are as follows.

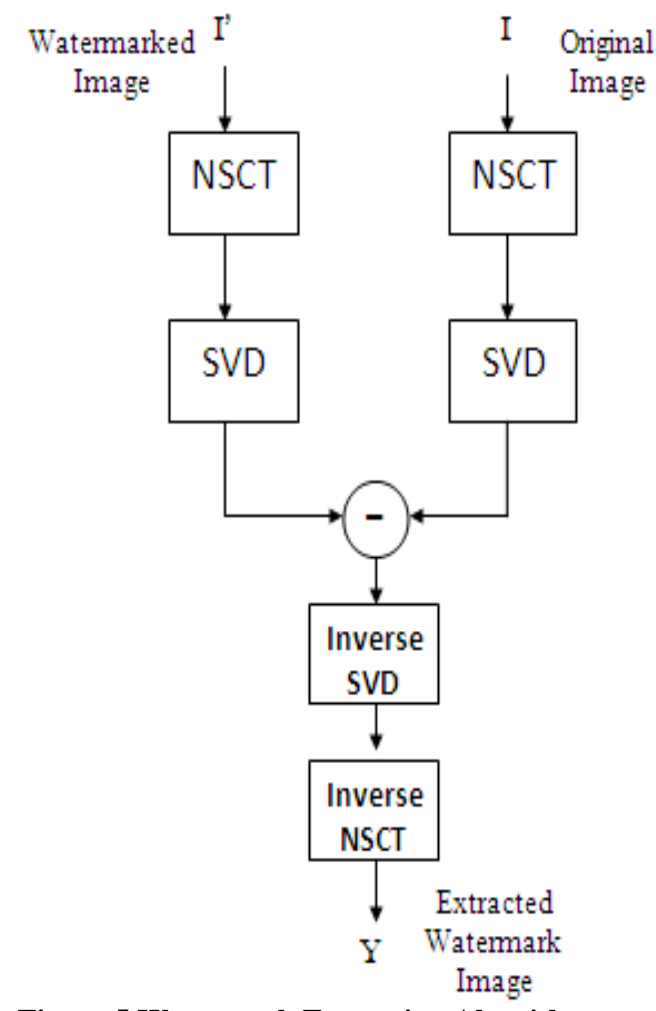

Figure 5 Watermark Extraction Algorithm

Step1: Apply NSCT to the watermarked image to decompose into sub bands.

Step2: Apply SVD to low frequency sub band of transformed watermarked image.

Step3: Extract the singular values from low frequency sub band of watermarked and original image $i, e$

$\lambda_{\mathrm{W}}=\left(\lambda_{\mathrm{I}},-\lambda_{\mathrm{I}}\right) / \alpha \quad$ Where $\lambda_{\mathrm{I}}$ is singular value of watermarked image.

Step4: Apply inverse SVD to obtain low frequency coefficients of transformed watermark image using Step 3.

Step5: Apply inverse NSCT using the coefficients of the low frequency sub band to obtain the gray scale Watermark image.

\section{EXPERIMENTAL RESULTS}

In the experiments, we use the standard grayscale "Lena.jpg" of size 512 X 512 as original image as shown in the Figure 6 and grayscale "Cameraman.jpg" of size 256 X 256 as watermark as shown in Figure 7. The results show that there are no perceptibly visual degradations on the watermarked image shown in Figure 8 with a PSNR of $37.6102 \mathrm{~dB}$. Extracted watermark without attack is shown in Figure 9 with NCC unity. MATLAB 7.6 version is used for testing the robustness of the proposed method. The proposed algorithm is also applied for different original images such as “Peppers.jpg","Baboon.jpg"."rice.jpg,",Barbara.jpg" and "Zoneplate.jpg" as in Table 1 and it is observed that there are no visual degradations on the respected watermarked images. For all the different original test images, the watermark is effectively extracted with unity NCC. Various intentional and non-intentional attacks are tested for robustness of the proposed watermark algorithm includes 
JPEG,JPEG2000compressions,Low pass filtering, Rotation, Histogram Equalization ,Median Filtering, Alpha Mean, Gray Scale Inversion ,Salt \&Pepper Noise, Soft Thresholding ,Weiner Filtering, Gamma Correction, Gaussian Noise, Rescaling ,Sharpening ,Blurring ,Contrast Adjustment ,Automatic and Manual cropping, Int Thresholding ,Dilation, Mosaic, Bit Plane Removal and Row Colum Copying.

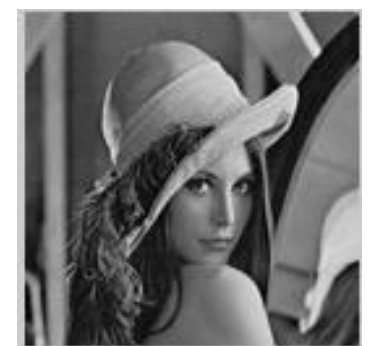

Figure 6:Original image"Lena"

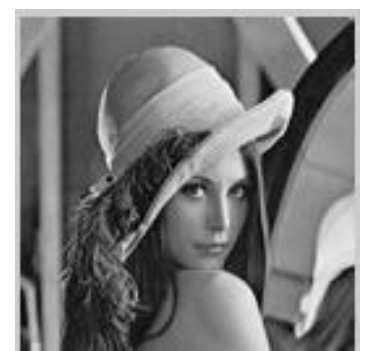

Figure 8: Watermarked "Lena" PSNR= 37.6102

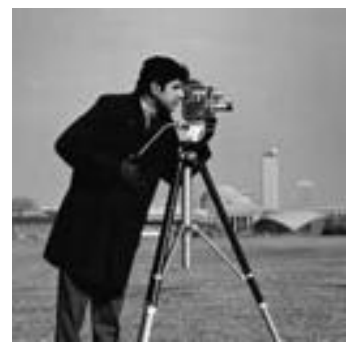

Figure 7:Watermark image-"Cameraman"

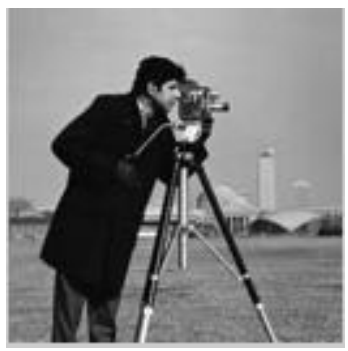

Figure9:Extracted Watermark Ncc $=1$
The proposed algorithm is compared with Emir Ganic and Ahmet M.Eskicioglu's paper [2] in which the watermarking is done by using DWT-SVD hybrid algorithm and the PSNR is reported as $34.42 \mathrm{~dB}$ and the No of attacks tested are only 12 . In our proposed scheme the PSNR obtained is $37.6102 \mathrm{~dB}$ and watermark image can survive up to 24 attacks compared to Emir Ganic and Ahmet M.Eskicioglu,as shown in Table2 and Table 3.

In Table 2 the normalized correlation coefficient values for different attacks are shown with extracted watermark Y and attacked watermarked image I'. The quality and imperceptibility of watermarked image $\mathrm{I}^{1}$ is measured by using PSNR which can be obtained using eq. 3 [20] with respect to original image $\mathrm{I}$. The similarity of extracted watermark $(\mathrm{Y})$ with original watermark $(\mathrm{X})$ embedded is measured using NCC which is given in eq. (4) [21].

$$
P S N R=10 \log \left[\frac{\max (I(i, j))^{2}}{\sum_{N, M}\left(I^{\prime}(i, j)-I(i, j)\right)^{2}}\right]
$$

Normalized Correlation Coefficient

$$
\mathrm{Ncc}=\frac{\sum_{m} \sum_{n}\left(A_{m n}-\bar{A}\right)\left(B_{m n}-\bar{B}\right)}{\sqrt{\left(\sum_{m} \sum_{n}\left(A_{m n}-\bar{A}\right)^{2}\right)\left(\sum_{m} \sum_{n}\left(B_{m n}-\bar{B}\right)^{2}\right)}}
$$

Table 1: watermarked and Extracted watermark with PSNR and NCC for different original images

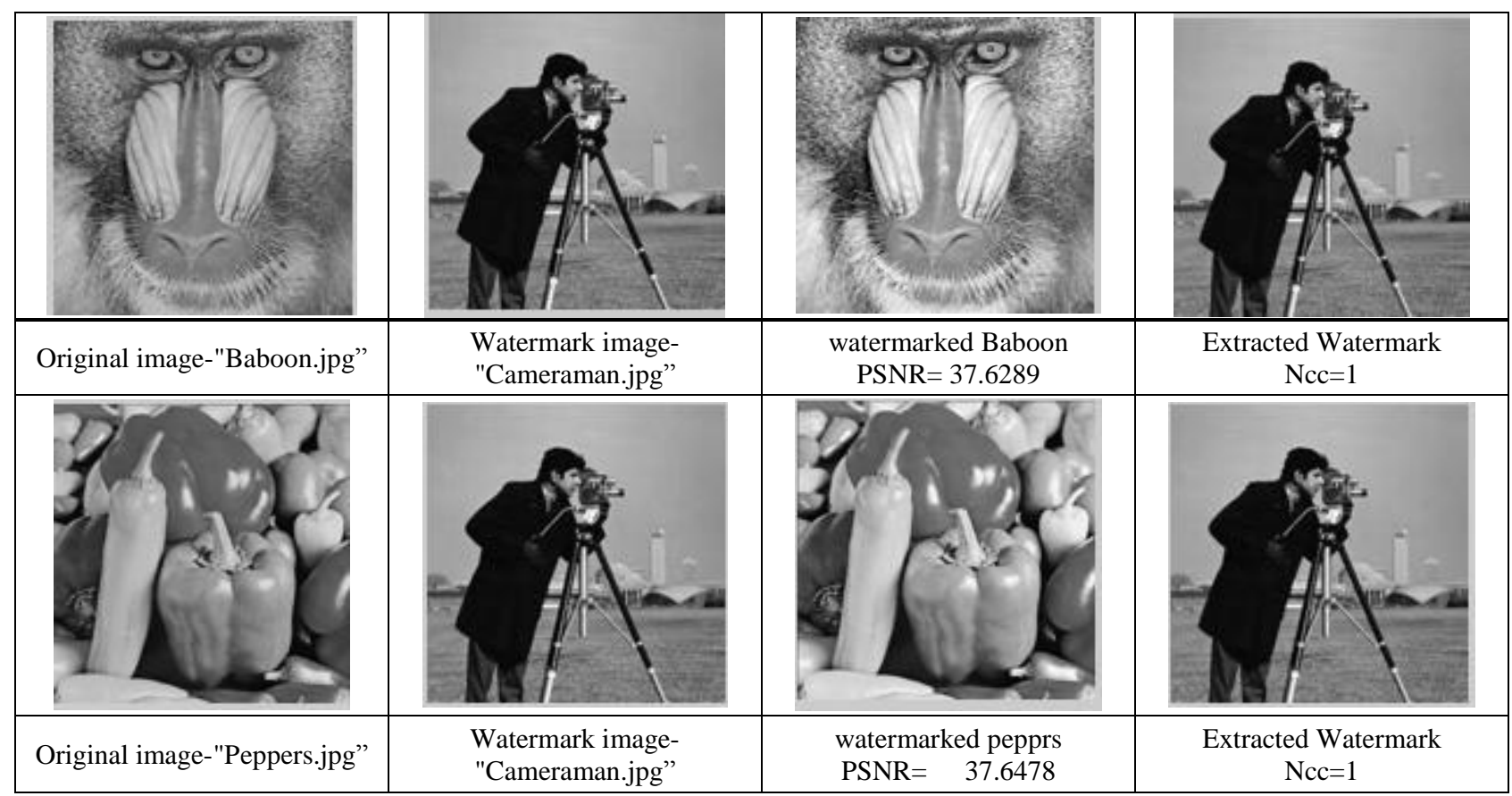




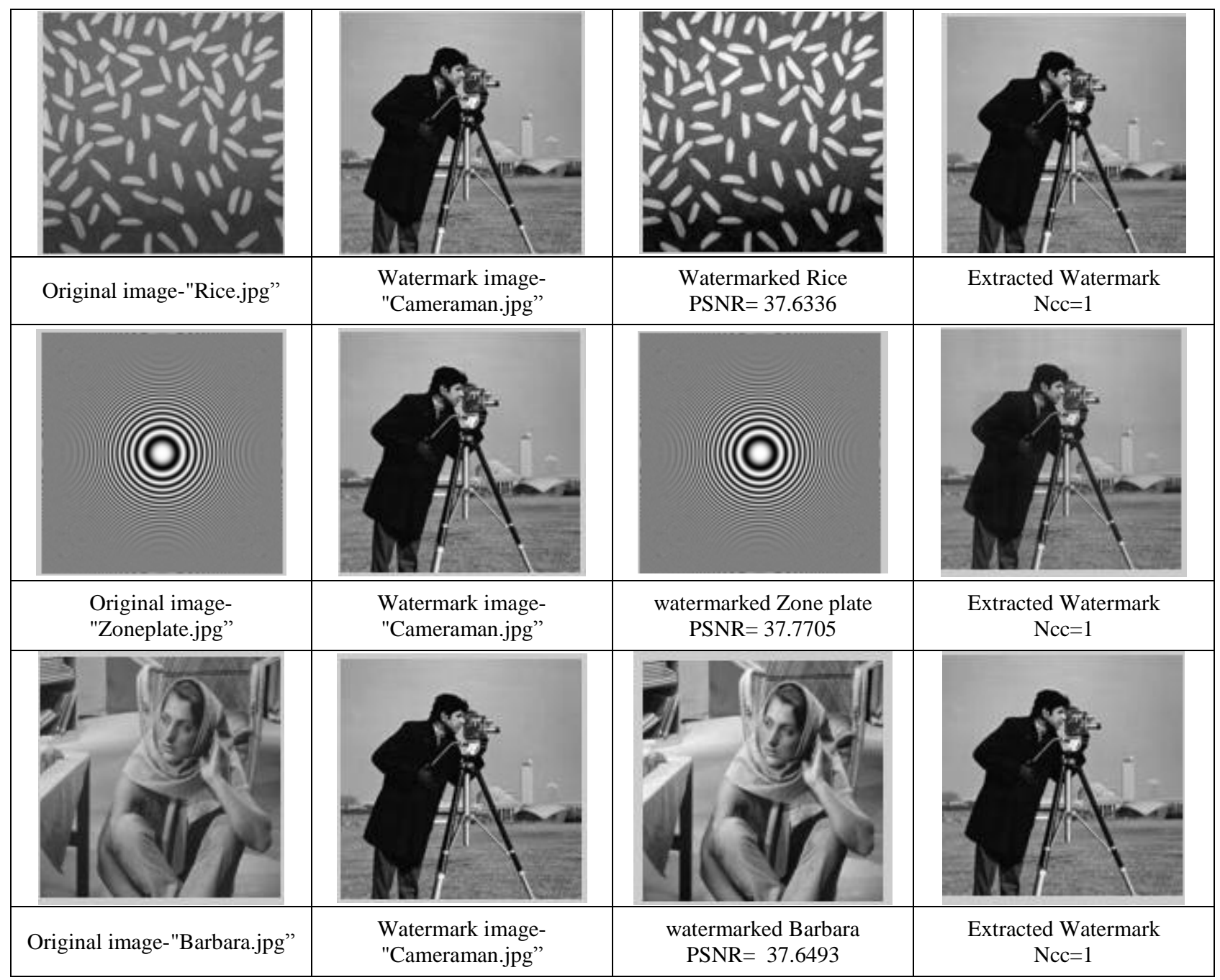

Table 2: Extracted watermarks with NCC for different attacks along with attacked watermarked image.

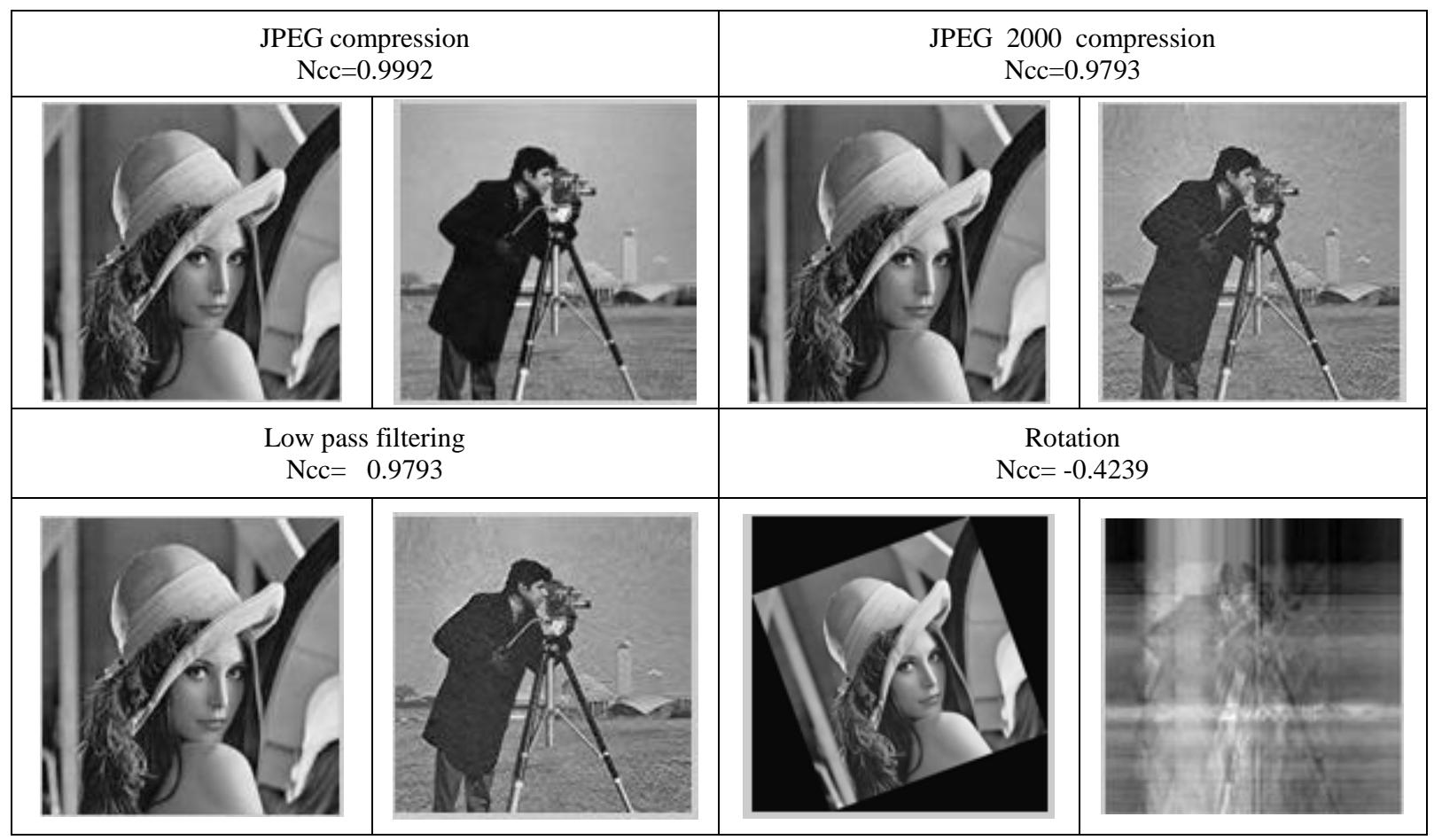




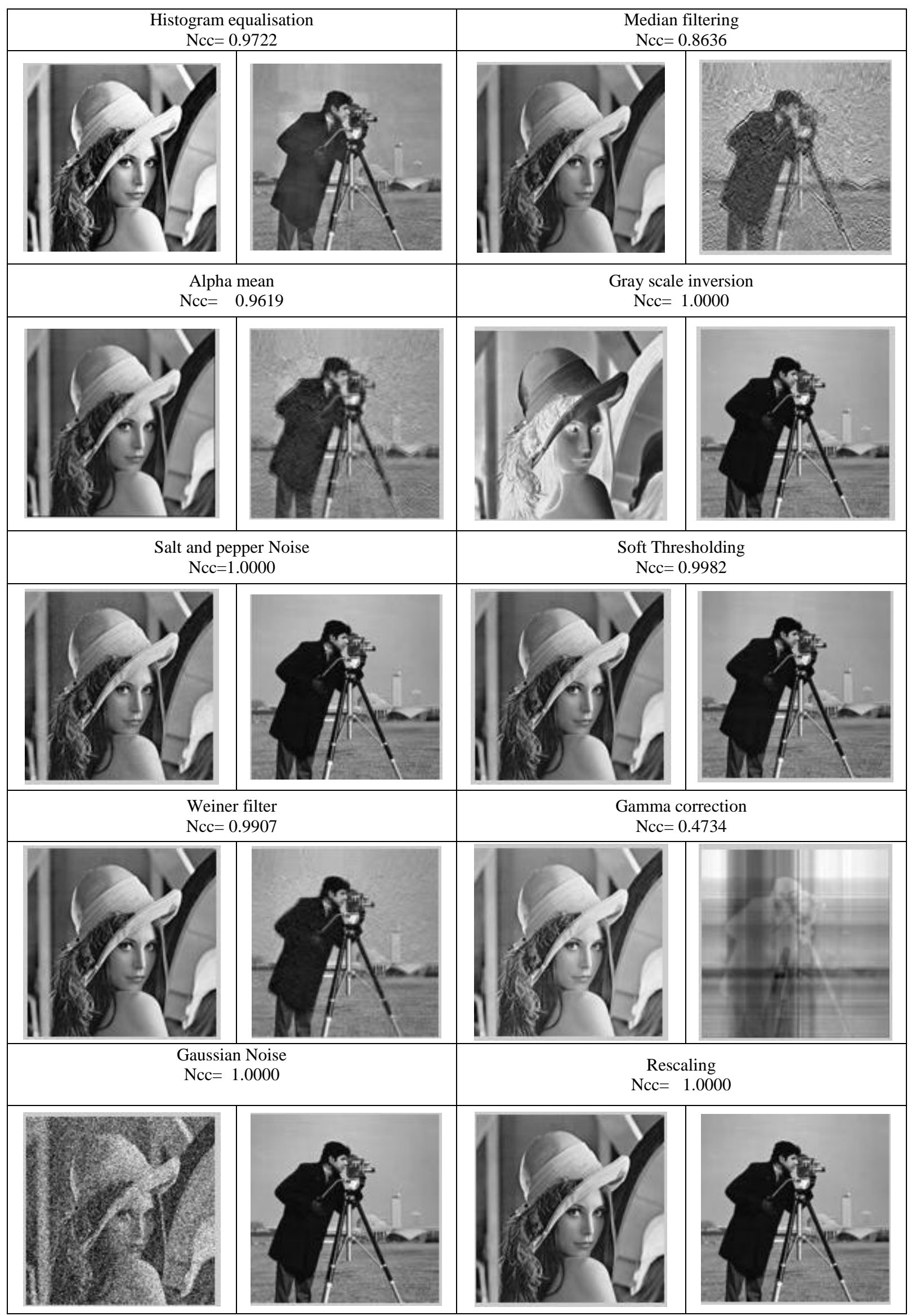




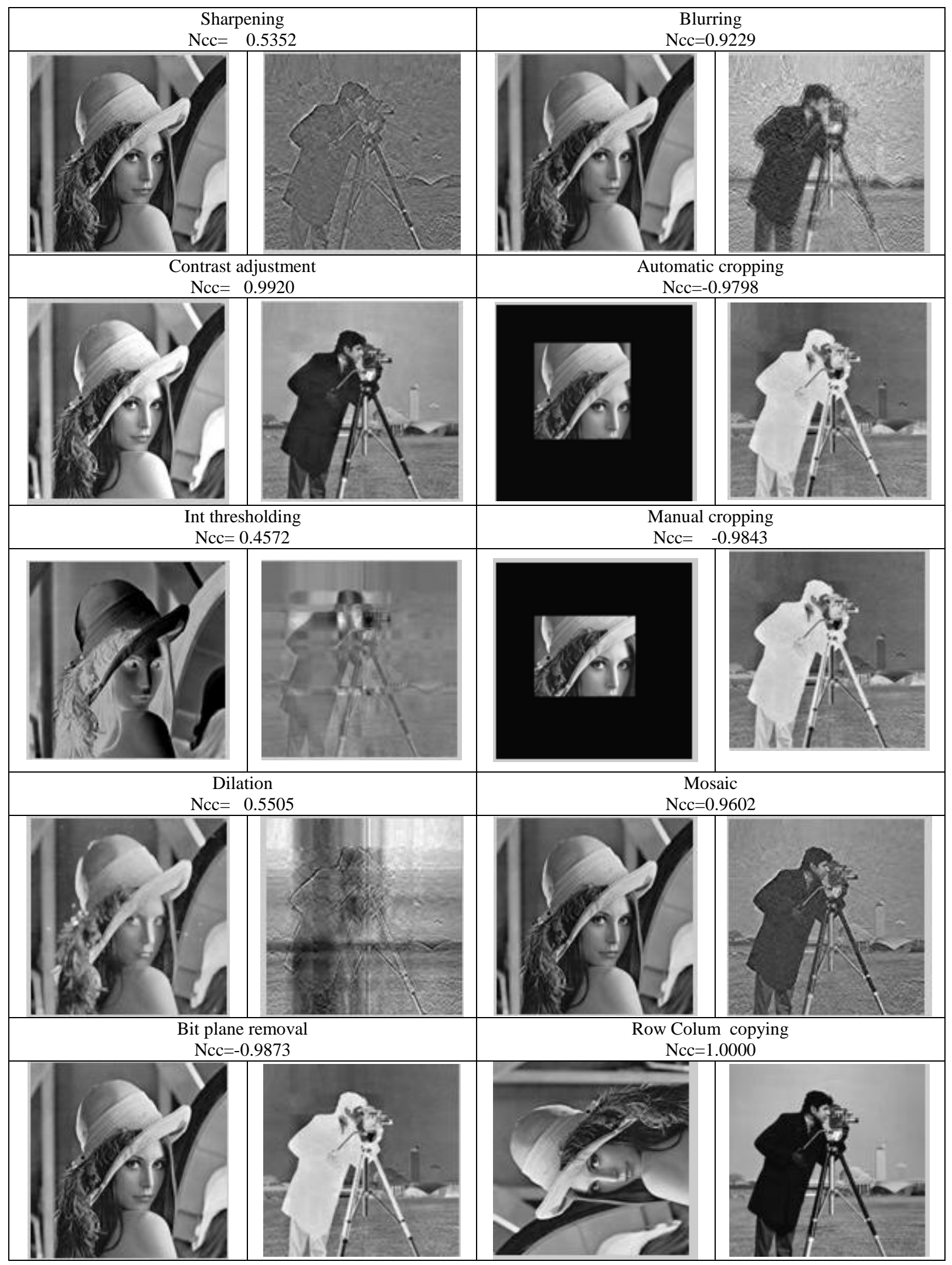

Table 3 Comparison of proposed method with Emir Ganic and Ahmet M.Eskicioglu's algorithm

\begin{tabular}{|c|c|c|}
\hline Characteristic & Proposed method & Emir Ganic and Ahmet M.Eskicioglu \\
\hline PSNR in DB & 37.6102 & 34.42 \\
\hline No of attacks tested & 24 & 12 \\
\hline
\end{tabular}


We also tested and compared the robustness to various attacks of the proposed method with simple singular value decomposition and with hybrid algorithms includes discrete wavelet transform and singular value decomposition, Contourlet transform and singular value decomposition as given in table 4 by taking gray scale "lena.jpg" of size $512 \mathrm{x}$ 512 as original image and gray scale "cameraman.jpg" of size $256 \times 256$ as watermark. The table 4 shows that proposed algorithm performs better for 16 attacks than that of other algorithms.

Table 4: Comparison of NCC of various attacks for different algorithms

\begin{tabular}{|c|c|c|c|c|c|}
\hline $\begin{array}{l}\text { S. } \\
\mathbf{N} \\
\mathbf{0}\end{array}$ & Attack & SVD & $\begin{array}{c}\text { DWT+ } \\
\text { SVD }\end{array}$ & $\begin{array}{l}\text { CT+ } \\
\text { SVD }\end{array}$ & $\begin{array}{c}\text { NSCT+ } \\
\text { SVD }\end{array}$ \\
\hline 1 & $\begin{array}{c}\text { Jpeg } \\
\text { compression }\end{array}$ & 0.8772 & 0.9992 & 0.9992 & 0.9992 \\
\hline 2 & $\begin{array}{c}\text { Jpeg2000 } \\
\text { compression }\end{array}$ & 0.8853 & 0.9501 & 0.9492 & 0.9793 \\
\hline 3 & $\begin{array}{l}\text { Low pass } \\
\text { filtering }\end{array}$ & 0.6197 & 0.9743 & 0.9681 & 1.0000 \\
\hline 4 & Rotation $\left(20^{\circ}\right)$ & 0.2510 & 0.2208 & 0.1792 & 0.2819 \\
\hline 5 & Auto cropping & -0.9508 & -0.9471 & 0.5975 & -0.9817 \\
\hline 6 & $\begin{array}{c}\text { Histogram } \\
\text { equalization }\end{array}$ & 0.9505 & 0.9537 & 0.8238 & 0.9722 \\
\hline 7 & $\begin{array}{l}\text { Median } \\
\text { filtering } \\
\end{array}$ & 0.5557 & 0.9602 & 0.9545 & 0.8636 \\
\hline 8 & Alpha mean & 0.8949 & 0.9458 & 0.9566 & 0.9619 \\
\hline 9 & $\begin{array}{l}\text { Gray scale } \\
\text { inversion }\end{array}$ & 0.9868 & 0.9868 & 0.9874 & 1.0000 \\
\hline 10 & $\begin{array}{c}\text { Salt and } \\
\text { pepper noise }\end{array}$ & 0.2131 & 0.9458 & 0.9507 & 1.0000 \\
\hline 11 & $\begin{array}{c}\text { Int- } \\
\text { thresholding }\end{array}$ & 0.4422 & 0.4456 & 0.4466 & 0.4572 \\
\hline 12 & $\begin{array}{c}\text { Soft } \\
\text { thresholding }\end{array}$ & 0.9982 & 0.9982 & 0.9981 & 0.9982 \\
\hline 13 & $\begin{array}{l}\text { Weiner } \\
\text { filtering }\end{array}$ & 0.0185 & -0.5727 & 0.7163 & 0.7794 \\
\hline 14 & $\begin{array}{c}\text { Gamma } \\
\text { correction }\end{array}$ & 0.5004 & 0.5030 & 0.0118 & 0.4734 \\
\hline 15 & $\begin{array}{l}\text { Gaussian } \\
\text { noise }\end{array}$ & 0.2590 & 0.9755 & 0.8414 & 1.0000 \\
\hline 16 & Rescaling & 1.0000 & 1.0000 & 1.0000 & 1.0000 \\
\hline 17 & Sharpening & 0.2440 & 0.6172 & 0.6137 & 0.5352 \\
\hline 18 & Blurring & 0.6306 & 0.9763 & 0.9693 & 0.9229 \\
\hline 19 & $\begin{array}{c}\text { Contrast } \\
\text { adjustment }\end{array}$ & 0.9997 & 0.9997 & 0.9864 & 0.9920 \\
\hline 20 & Mosaic & 0.9188 & 0.9702 & 0.9704 & 0.9602 \\
\hline 21 & $\begin{array}{l}\text { Manual } \\
\text { cropping }\end{array}$ & -0.7885 & -0.9530 & 0.5101 & -0.9806 \\
\hline 22 & Dilation & 0.5384 & 0.4058 & 0.0300 & 0.5505 \\
\hline 23 & $\begin{array}{l}\text { Bit plane } \\
\text { removal }\end{array}$ & -0.9689 & -0.9648 & -0.9742 & -0.9873 \\
\hline 24 & $\begin{array}{l}\text { Row column } \\
\text { copying }\end{array}$ & 0.9987 & 0.9997 & 1.0000 & 1.0000 \\
\hline
\end{tabular}

\section{CONCLUSION}

In this paper, a novel, yet simple, hybrid nonsubsampled contourlet domain SVD based watermarking scheme for image copyright protection is proposed, where the singular values of low frequency sub band coefficients of watermark image are embedded on the singular values of low frequency sub band coefficients of original image with an appropriate scaling factor. The proposed algorithm preserves high perceptual quality of the watermarked image and shows an excellent robustness to attacks like JPEG, JPEG2000 compressions, Low pass filtering, Histogram equalization, Gray scale inversion, Salt and Pepper Noise, Soft Thresholding, Weiner Filtering, Gaussian Noise, Rescaling and Contrast adjustment. This algorithm is quite resilient to Rotation, Median filtering, Alpha mean, Gamma correction,
Sharpening, Blurring, Cropping, Dilation, Int thresholding, Mosaic and bit plane removal attacks. The proposed algorithm achieve s higher PSNR when compared with Emir Ganic and Ahmet M.Eskicioglu's paper. It demonstrates that nonsubsampled contourlet transform domain performs better than wavelet domain. The proposed algorithm is also tested for different original images and respective watermarked images are obtained without any visual degradation.

\section{REFERENCES}

[1] Santi P.Maity, Prasun Nandy, Tirtha S.Das and Maly K.Kundu, "Robust image watermarking using multiresolution analysis", IEEE INDIA Annual Conference 2004,INDICON 2004

[2] Emir Ganic and ahmet M. Eskicioglu “ Robust embedding of visual watermarks using discrete wavelet transform and singular value decomposition Journal. Of Electron. Imaging, Vol. 14, 043004 (2005); doi:10.1117/1.2137650 Published 12 December 2005

[3]

Alexander Sverdlov, Scott Dexter and Ahmet M.Eskicioglu "Robust DCT_SVD domain image Watermarking for copyright protection: embedding data in all frequencies".

[4] C.Venkata Narasimhulu and K.Satya Prasad, "A hybrid watermarking scheme using contourlet Transform and Singular value decomposition" IJCSNS: International Journal of Computer Science and Network Security, Vol.10,No.9, September 2010.

[5] Minh N. Do, and Martin Vetterli, "The Contourlet Transform: An Efficient Directional Multiresolution Image Representation" IEEE transaction on image processing,vol 14,issue no 12,pp 2091-2106,Dec 2005

[6] Elham salahi ,M.Shahram Moin and Ahmad salahi "A new Visually Imperceptible and Robust Image Water marking Scheme in contourlet Domain" International conference on intelligent information hiding and multimedia signal processing, 2008 .

[7]

Jianping Zhou; Cunha, A.L, M.N.Do, "Nonsubsampled contourlet transform construction and application in enhancement" IEEE Trans. Image Proc Sept. 2005

[8]

Arthur L. Cunha, J. Zhou, and M. N. Do, "Nonsubsampled contourlet transform: filter design and applications in denoising" IEEE international conference on image processing, September 2005.

[9] V.I.Gorodetski L.J.Popyack, and V.Samoilov, "SVDbased approach to transparent embedding data into digital images," in proc. int. workshop, MMM-ACNS, St.Peterburg, Russia, May 2001,pp.263-274.10. R.Liu and T.Tan, "An SVD-Based Watermarking scheme for protecting rightful ownership," IEEE Trans. Multimedia, vol.4, no.1, pp.121-128, Mar.2002.

[11] Paul Bao and Xiaohu Ma "Image adaptive watermarking using wavelet domain singular value decomposition" IEEE Transaction on circuit and system for video technology, vol.15, no.1, January 2005.

[12] A. L. Cunha, J. Zhou, and M. N. Do, "The Nonsubsampled contourlet transform: theory, design and applications,” IEEE Trans. Image Proc., May 2005. 
[13] A. L. Cunha, J. Zhou, and M. N. Do, "The Nonsubsampled contourlet transform: theory, design and applications," IEEE Trans. Image Proc., vol.15, no.10, October 2006.

[14] Xiang Yang Wang, Yi-Ping Yang and Hong-Ying Yang, "A novel nonsubsampled contourlet domain Image watermarking using Support Vector Regression", Journal of Optics: A Pure and Applied Optics, September 2009.

[15] M.J.Shensha,'The discrete Wavelet Transform: Wedding the A Trous and Mallat algorithms", IEEE Trans, vol 40, no 10, Pp.2464-2482, Oct 1992.

[16] R.H.Bamberger and M.J.T.S,ith. "Afilter bank for the directional decomposition of images: Theory and Design", IEEE Trans .Signal Processing vol.40,no.4,pp.882-893,Apr 1992

[17] B.Chandra Mohan and S.Srinivas Kumar "A Robust Image watermarking scheme using Singular value decomposition" Journal of Multimedia,Vol3,NO:1,May 2008 .

[18] Ke-Feng He,Jun Gao,Liang-Mei Hu, "Watermarking for images using the HVS and SVD in the Wavelet Domain", Procedings fo 2006 IEEE International on Mechatronics and Automation June 25 to 28,2006, Luoyang,Chaina.

[19] Salwa A.K.Mostafa, A.S.Tolba, F.M.Abdelkader, Hisham M.Elhindy, "Video Watermarking Scheme based on Principal Component Analysis and Wavelet Transform", IJCSNS: International Journal of Computer Science and Network Security, Volume9, No: 8, August 2009.
[20] Ashraf. K. Helmy and GH.S.El-Taweel "Authentication Scheme Based on Principal Component Analysis for Satellite Images" International Journal of Signal Processing, Image Processing and Pattern Recognition Vol. 2, No.3, September 2009.

[21] Matlab 7.6 version ,Image Processing Tool Box.

\section{AUTHORS PROFILE}

\section{C.V Narasimhulu}

$\mathrm{He}$ received his Bachelor degree in Electronics and Communication Engineering from S.V. University, Tirupati, India in 1995 and Master of technology in Instruments and Control Systems from Regional Engineering College Calicut, India in 2000. He is currently pursuing the Ph.D degree in the department of Electronics and Communication Engineering from Jawaharlal Nehru Technological University Kakinada, India. He has more than 15 years experience of teaching under graduate and post graduate level. He is interested in the areas of signal processing and multimedia security.

\section{K.Satya Prasad}

He received his Ph.D degree from IIT Madras, India. He is presently working as professor in the department of Electronics and Communication Engineering, JNTU college of Engineering Kakinada and Rector of Jawaharlal Nehru Technological University, Kakinada, India. He has more than 30 years of teaching and research experience. He published 30 research papers in international and 20 research papers in National journals. He guided 8 Ph.D theses and $20 \mathrm{Ph} . \mathrm{D}$ theses are under his guidance. His area of interests includes Digital Signal and Image Processing, Communications, Adhoc networks...etc. 